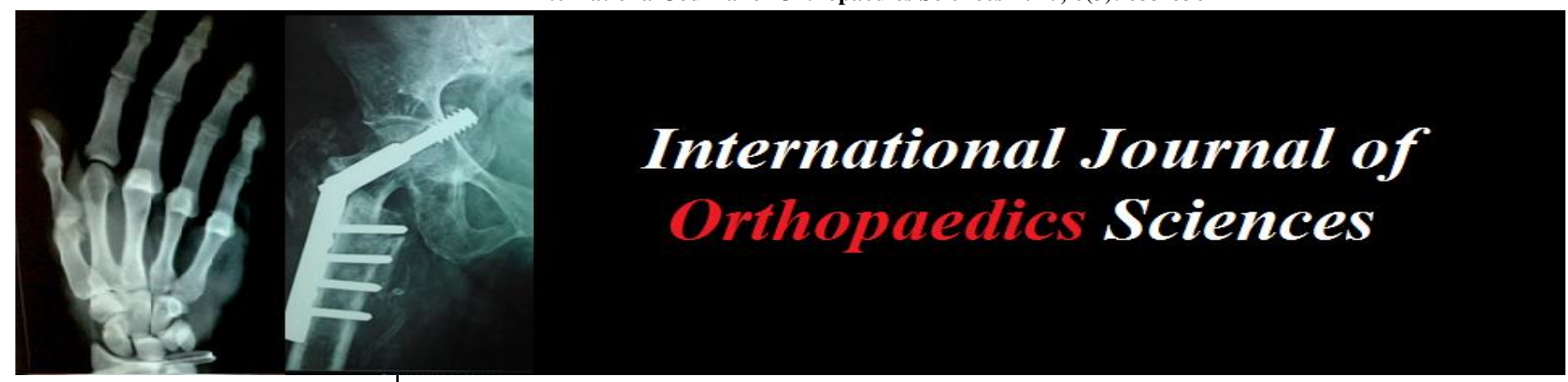

E-ISSN: 2395-1958

P-ISSN: 2706-6630

IJOS 2020; 6(3): 633-636

(C) 2020 IJOS

www.orthopaper.com

Received: 14-05-2020

Accepted: 16-06-2020

\section{Akhil Bansal}

Associate Professor, Department of Orthopaedics, Gandhi Medical College, Bhopal, Madhya

Pradesh, India

Sourabh Alawa

Resident, Department of Orthopaedics, Gandhi Medical

College, Bhopal, Madhya

Pradesh, India

\section{Deependra Sonkar}

Associate Professor, Department of Orthopaedics, Gandhi Medical College, Bhopal, Madhya Pradesh, India

\section{Arvind Karoria}

Resident, Department of Orthopaedics, Gandhi Medical College, Bhopal, Madhya

Pradesh, India

\section{Anshul Khare}

Resident, Department of

Orthopaedics, Gandhi Medical

College, Bhopal, Madhya

Pradesh, India

\section{Sanjiv Gaur}

Professor and Head of

Department, Department of Orthopaedics, Gandhi Medical College, Bhopal, Madhya

Pradesh, India
Corresponding Author: Sourabh Alawa Resident, Department of Orthopaedics, Gandhi Medical College, Bhopal, Madhya Pradesh, India

\section{Supracutaneous locking compression plate as an external fixator in compound metaphyseal and diaphyseal fractures of tibia}

\author{
Akhil Bansal, Sourabh Alawa, Deependra Sonkar, Arvind Karoria, \\ Anshul Khare and Sanjiv Gaur
}

DOI: $\underline{\text { https://doi.org/10.22271/ortho.2020.v6.i3j.2263 }}$

\begin{abstract}
Background: Most of lower leg external fixation are bulky and cumbersome and provides only temporary bony stabilization. Anatomically pre-contoured supracutaneous LCP has been utilized by many authors as an external fixator in patients with metaphyseal and diaphyseal fractures of tibia.

Aim: The aim of this study is to analyse the end result of Supracutaneous LCP as a definitive external fixation and its effect on rate of union in metaphyseal and diaphyseal fractures.

Materials and methods: A complete of 30 patients of compound fractures of tibia underwent "supracutaneous plating" of the tibia using an LCP. Average age was 44 years. Regular screw tract dressings were done. Average period of follow-up was 13 months.

Results: The plate was kept ex situ for a period of 24-30 weeks. Proximal metaphysis, diaphysis and distal metaphysis united in 14 weeks, 20 weeks and 16 weeks respectively. Total of 30 patients were operated for Supracutaneous LCP of which one complicated with Infected Non-union resulting in implant removal and other complicated with Non-union which later underwent internal fixation.

Conclusion: In compound fractures of tibia supracutaneous LCP are often used as a definitive external fixator because it gives good results especially for metaphyseal fractures and fewer complication rates, with Adequate stability. Advantageous effect of LCP in facilitating wound healing, cosmetic surgery procedure. Because it does not strike the contralateral leg therefore cosmetically acceptable and functional while ambulation. Because of nonoverlapping of implants fracture healing is easily assessed on $\mathrm{x}$ rays
\end{abstract}

Keywords: Supracutaneous LCP, compound tibial fractures, external fixation by plate, locking compression plate

\section{Introduction}

In compound fractures of tibia, debridement followed by fracture fixation is that the usually followed two stage treatment protocol ${ }^{[1,2]}$ within the management. The soundness of the fracture after debridement will prevent infection and promote wound healing. So, temporary fracture stabilization by external fixation ${ }^{[3,4]}$ is advocated. The external fixator frames used in management of fractures of tibia are large and bulky which hinders the movement of both the legs during the gait cycle. They cannot be hidden under clothes and are not cosmetically well looking. Thus, these devices demand more compliance from the patient.

Open fractures Management ${ }^{[5,6]}$ using Locking compression plate as an external fixation device is described earlier. The Zespol System ${ }^{[1]}$ was the earliest design for external plate fixation, which involves multiple nuts and washers, which cause surgeons receptive to discourage this method.

Since the innovation of locking plate which has lesser moving parts as compared traditional parts there has been renewed interests in this technique ${ }^{[7,8,9,10,11]}$.

External fixator has been used widely in the treatment of open fractures, septic arthritis, nonunions and as an aid in distraction osteogenesis. The application of LCP as an external fixator is described as "supracutaneous plating technique." [12, 13, 14, 15, 16, 17, 18] Similarly the plates as external fixators may also have also possible roles in metaphyseal and diaphyseal fracture union. In this study we try to analyse the outcome of anatomical precontoured locking plates 
as an external fixator device in 30 patients of open fractures of tibia.

\section{Material and methods}

An observational study of patients with compound injuries of tibia during a period of august 2017 to march 2019. Total 30 fractures were treated by Supracutaneous locking compression plate application. Their mean age was 45 years (24 to 66$)$.

\section{Aims and objectives}

To assess the functional outcome of Compound Tibial metaphyseal and diaphyseal fractures treated with Supracutaneous LCP and to check the complications of Compound fractures. Inclusion criterion are all fresh cases of open fracture of tibia from ${ }^{[5,6]}$ Gustilo Anderson type II to type IIIB metaphysio-diaphyseal Fractures. Exclusion criterion are pathological fractures, patients related to ipsilateral femur fractures, patients with neurological and vascular deficit and open fracture without adequate soft tissue coverage.

\section{Operative procedure}

Anaesthesia

Spinal anaesthesia is used, the involved limb is prepped and draped with standard aseptic precaution.

\section{Technique}

- As per the grade of compound fracture, pre antibiotic were administered and intervention is dole out without tourniquet so as to allow adequate antibiotic perfusion.

- thorough debridement and wound wash is given and fracture alignment achieved before wound closure (usually open wound is sutured in single layer before placing the plate because it may hinder accessibility of wound).

- Locking compression plate of appropriate length is chosen in line with the position of fracture.

- It is preferred to use at least 3 to 4 screws in both the proximal and distal fragments of the fracture.

- Plate is initially fixed to proximal and distal fragments with the assistance of $\mathrm{k}$ wire after fracture reduction under fluoroscopy guidance.

- LCP is placed at such distance from underlying skin such providing adequate space for swelling and wound care and adequate mechanical stabilization.

- Usually a custom made spacer helps to keep equal distance of plates.

- Bi cortical screw fixation is completed with locking screw and plate fixed.

- After achieving satisfactory reduction subsequent holes are drilled through stab incisions using drill sleeves. It is preferable to put screws first in distal fragment followed by proximal fragment. The size, position and orientation of screws are confirmed under fluoroscopy.

- For comminuted tibial fractures, reduction of length and alignment were achieved by traction and percutaneous manipulation with $\mathrm{k}$ wires under fluoroscopy guidance.

- For the distal tibia, a minimum of four screws $(4.5 \mathrm{~mm})$ proximally and three to four screws $(3.5 \mathrm{~mm})$ distally is suggested. Stab incision were made on intact soft tissues and locking drill guides were placed. Successive holes were drilled over locking drill-guides. Screws were placed first in distal fragment and then in proximal fragment after ensuring adequate reduction.
CASE 7/30

Fig. 1 A 34-year-old man presented with compound grade II fracture anteroposterior view showing distal tibia fracture of his right leg preoperatively $(\mathrm{a}, \mathrm{b})$ and postoperatively $(\mathrm{c}, \mathrm{d})$ and follow up after 16 weeks (e, f) and after implant removal at 22 weeks $(\mathrm{g}, \mathrm{h})$.
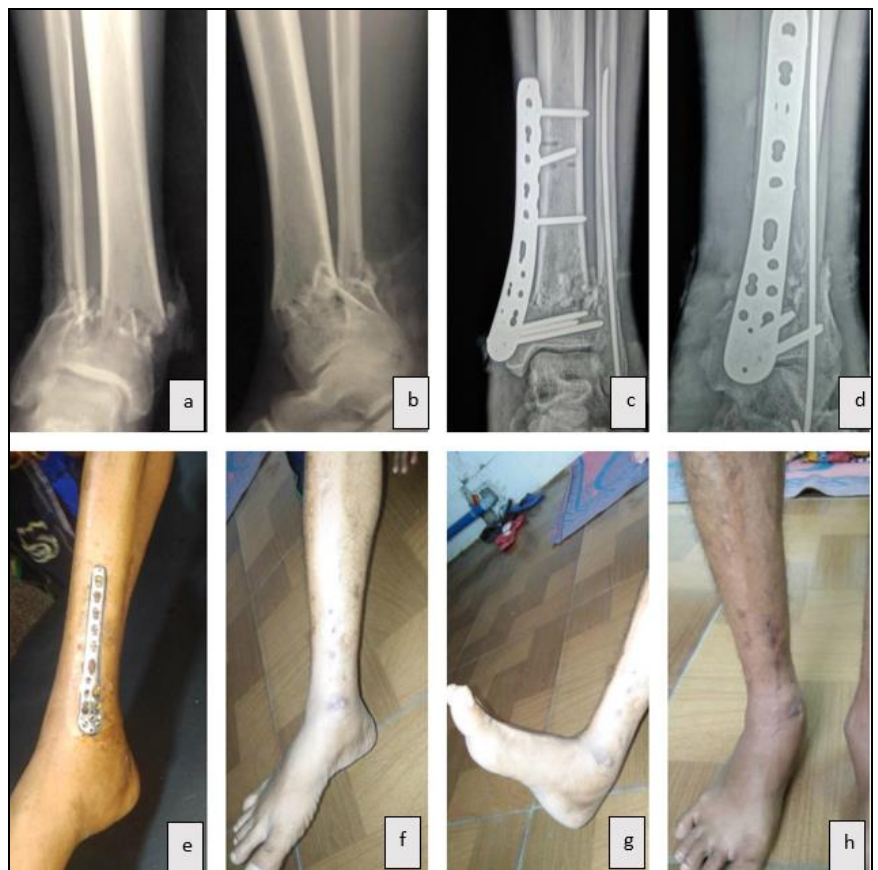

\section{CASE NO - 16/30}

Fig. 1 A 61-year-old man presented with compound grade IIIb, anteroposterior radiograph showing communited segmental fracture both bone right leg $(\mathrm{a}, \mathrm{b}, \mathrm{c})$, which was treated by supracutaneous metaphyseal LCP (d). Follow up after 3 months with complete wound healing with no screw site infection and full weight bearing (e). 7 months later, radiological union was achieved. Supracutaneous LCP was removed after 8 months and his ankle showed a good function and there were no residual signs of infection(f).
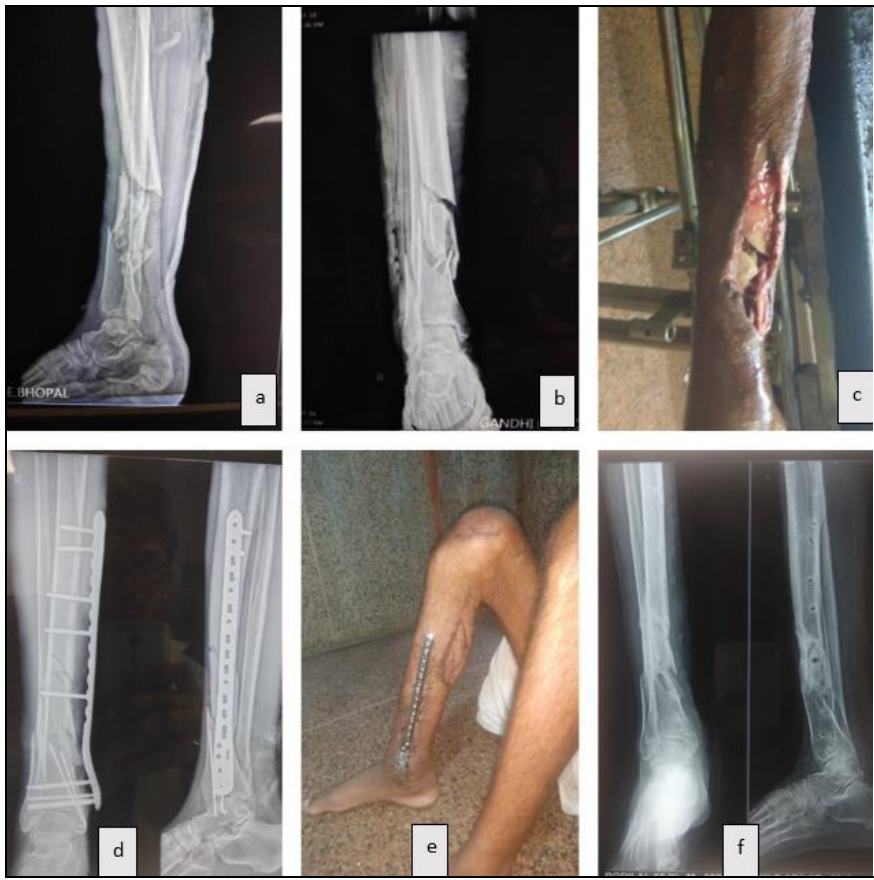

Screw tract and wound dressing ${ }^{[19]}$ were done. Knee range of movement exercises and non-weight bearing walking was 
allowed from immediate post-operative day as soon as patient was comfortable. Toe touch partial weight bearing were allowed after 4 weeks, which continued for next 6 weeks. Consistent with the steadiness of fixation and healing of fracture, complete weight bearing were started.

\section{Results}

We prospectively evaluated 30 cases of fresh compound tibial fractures. The mean duration of surgery was 42 minutes range (35 minutes to 60 minutes), The mean fracture healing time was 14 week (range, 12-18 weeks) for proximal tibia, 24 weeks (range, 18-26 weeks) for tibial diaphysis / multi segmental fractures, 18 weeks (range, 14-20 weeks) for distal tibia. Once biplaner radiographic cortical bridging was observed, full weight bearing for 1 month before implant removal was advised. 19 of 30 cases were males and rest 11 were females. Average age was found to be 45 years with a range of 24 to 66 years. According to the Gustilo Anderson classification ${ }^{[5,6]}$ the fractures were classified as 7 cases of grade II, 14 cases of grade IIIa and 9 cases of grade IIIb. Total of 30 patients were operated for Supracutaneous LCP of which, one complicated with Infected Non union causing implant removal and other complicated with Non union which undergoes internal fixation by tibial interlock nailing later on. Superficial pin tract infection in one patient which got healed with regular dressings. According to the Johner and Wruhs criteria ${ }^{[20]}$ Excellent in $75 \%$, Good in $18 \%$, Poor in $7 \%$. and Oleaur and Molander score 3 was Excellent in $79 \%$, Good in $14 \%$, Poor in $7 \%$ at the end of the study. Representative cases for distal tibial fractures (Figure 1, a-h) multi segmental diaphyseal fracture (Figures2, a-f).

\section{Discussion}

In Contrast with traditional external fixators, which are often heavy, locking plates have a low profile and thus are less likely to strike the contralateral lower leg in the swingthrough phase of either leg during ambulation ${ }^{[21]}$.

Under stockings the plate may be well concealed because it is placed close to the skin, enabling patients to steer while wearing trousers. The anteromedial aspect of the tibia are often clearly palpated, facilitating fast and accurate insertion of screws with less risk of neurovascular injuries. Bicortical screws were utilized in this study. All of those features increase the steadiness of the construct ${ }^{[22]}$. All of the patients eventually achieved fracture healing. Conventional treatment for tibial fractures includes plating or nailing. Nevertheless, the submuscular or subcutaneous plates could also be prominent under the skin or muscles and may cause soft tissue problems [23]. Additionally, anterior knee pain is often reported after antegrade tibial nailing. One stage supracutaneous LCP fixation decreases both costs and surgical injuries ${ }^{[24]}$. Fractures are anatomically reduced via a little incision without massive dissection. The LCP and screws were placed medially have less influence on muscle activity. Because stab incisions were used for screw insertion, and thus digging and tunnelling round the bone wasn't necessary, the possibility of infection might be decreased. With external plating, the chances of deep infection are reduced due to maintenance of the integrity of the soft tissue envelope. In contrast, surgery to withdraw an intramedullary nail or insitu locking plate fixation can lead to complications

\section{Conclusion}

Locking compression plate can be used as a definitive external fixator for compound tibial fractures as it gives good results and low complication rates, with satisfactory stability $[12,13,14,15,16,17,18,25]$. It also has the advantage of facilitating wound healing, plastic procedures. It is also cosmetically acceptable and non-cumbersome while mobilization as it does not strike the opposite leg. It allows easy assessment of fracture healing on $\mathrm{x}$ rays due to nonoverlapping of the implant. The use of LCPs as external fixators is not popular and there is little evidence in the literature ${ }^{[26]}$ but it has the advantage of less financial burden to rural and poor people with better embracing as early mobilization of joints are allowed with lesser complication.

Future prospects - Available literatures are studies on small sample size. Large studies are required for proving efficacy and understanding rare adverse effects.

\section{References}

1. Ramotowski W, Granowski R. Zespol. An original method of stable osteosynthesis. Clinical orthopaedics and related research. 1991; (272):67-75.

2. Ma CH, Tu YK, Yeh JH, Yang SC, Wu CH. Using external and internal locking plates in a two-stage protocol for treatment of segmental tibial fractures. Journal of Trauma and Acute Care Surgery. 2011; 1;71(3):614-9.

3. Mei ZF, Fan SW, Zhao FD, Wang CY, Liu JH, Shan Z. Locking plate external fixator for the treatment of middle and distal tibial fractures. Zhongguo gu shang= China journal of orthopaedics and traumatology. 2014; 27(6):458-60.

4. Zhao W, Cao Y, Shi LJ, Li T. Locking plate percutaneous external fixation for the treatment of pediatric tibial fractures. Zhongguo gu shang = China journal of orthopaedics and traumatology. 2014; 27(7):597-600.

5. $\mathrm{Rb} \mathrm{G}$, Anderson JT. Prevention of infection in the treatment of one thousand and twenty-five open fractures of long bones: retrospective and prospective analyses. J Bone Joint Surg Am. 1976; 58(4):453-8.

6. Gustilo RB, Mendoza RM, Williams DN. Problems in the management of type III (severe) open fractures: a new classification of type III open fractures. The Journal of trauma. 1984; 1;24(8):742-6.

7. Frigg R. Locking compression plate (LCP). An osteosynthesis plate based on the dynamic compression plate and the point contact fixator (PC-Fix). Injury. 2001; 1;32:63-6.

8. Kerkhoffs GM, Kuipers MM, Marti RK, der Werken V. External fixation with standard AO-plates: technique, indications, and results in 31 cases. Journal of orthopaedic trauma. 2003; 1;17(1):61-4.

9. Apivatthakakul T, Sananpanich K. The locking compression plate as an external fixator for bone transport in the treatment of a large distal tibial defect: a case report. Injury. 2007; 1;38(11):1318-25.

10. Kloen P. Supercutaneous plating: use of a locking compression plate as an external fixator. Journal of orthopaedic trauma. 2009; 1;23(1):72-5.

11. Woon CY, Wong MK, Howe TS. LCP external fixationexternal application of an internal fixator: two cases and a review of the literature. Journal of orthopaedic surgery and research. 2010; 5(1):1-6.

12. Patil SN. A prospective study of using a locking compression plate as external fixator for open distal tibial fractures. International Journal of Orthopaedics. 2018; $4(2): 42-7$. 
13. Rajasekaran S, Jayakumar B. Supracutaneous Locking Compression Plate for Grade II Compound Fracture Tibia: A Case Series. International Journal of Scientific Study. 2016; 1;4(2):74-6.

14. Prashanth PS et al. A Comparative Study of Uniplanar Unilateral External Fixation Versus Locking Plate as External Fixation in The Definitive Management of Open Fractures of Tibial Diaphysis in Adults. IOSR Journal of Dental and Medical Sciences. 2016; 15:50-52.

15. Ma CH, Wu CH, Jiang JR, Tu YK, Lin TS. Metaphyseal locking plate as an external fixator for open tibial fracture: clinical outcomes and biomechanical assessment. Injury. 2017; 1;48(2):501-5.

16. Luo $\mathrm{P}, \mathrm{Xu} \mathrm{D}, \mathrm{Wu} \mathrm{J}$, Chen $\mathrm{YH}$. Locked plating as an external fixator in treating tibial fractures: A PRISMAcompliant systematic review. Medicine. 2017; 96(49).

17. Thein WM, Mohamad Y, Lwin T, Htun AT. Supracutaneous plate: A comparable alternative to external fixator in the treatment of open fracture. International Journal of Orthopaedics. 2018; 4(2):959-62.

18. Patil SN. A prospective study of using a locking compression plate as external fixator for open distal tibial fractures. International Journal of Orthopaedics. 2018; $4(2): 42-7$.

19. Lau TW, Leung F, Chan CF, Chow SP. Wound complication of minimally invasive plate osteosynthesis in distal tibia fractures. International orthopaedics. 2008; 1;32(5):697.

20. Johner R, Wruhs O. Classification of tibial shaft fractures and correlation with results after rigid internal fixation. Clinical orthopaedics and related research. 1983; (178):725.

21. Tulner SA, Strackee SD, Kloen P. Metaphyseal locking compression plate as an external fixator for the distal tibia. International orthopaedics. 2012; 1;36(9):1923-7.

22. Kanchanomai C, Phiphobmongkol V. Biomechanical evaluation of fractured tibia externally fixed with an LCP. Journal of applied biomechanics. 2012; 1;28(5):587-92.

23. Qiu XS, Yuan H, Zheng X, Wang JF, Xiong J, Chen YX. Locking plate as a definitive external fixator for treating tibial fractures with compromised soft tissue envelop. Archives of orthopaedic and trauma surgery. 2014; $1 ; 134(3): 383-8$.

24. Zhang J, He X, Li M, Yu Y, Zhu L. Treatment of segmental tibial fractures with super cutaneous plating. Orthopedics. 2014; 1;37(8):e712-6.

25. Wu G, Luo X, Tan L, Lin X, Wu C, Guo Y et al. Comparison study on locking compress plate external fixator and standard external fixator for treatment of tibial open fractures. Zhongguo xiu fu chong jian wai ke za zhi= Zhongguo xiufu chongjian waike zazhi= Chinese journal of reparative and reconstructive surgery. 2013; 27(11):1291-5.

26. Zhang JW, Ebraheim NA, Li M, He XF, Schwind J, Zhu LM et al. Distal tibial fracture: an ideal indication for external fixation using locking plate. Chinese Journal of Traumatology. 2016; 1;19(2):104-8. 